\title{
Discussion of applications of molecular biological techniques to the field of microorganism in environmental engineering
}

\author{
Xinlei $\mathrm{Li}^{1, a}$ \\ ${ }^{1}$ Department of biological science, $\mathrm{Xi}$ ' an Jiao tong-Liverpool University, Jiangsu, Suzhou, \\ 215123, China \\ a915867466@qq.com
}

\begin{abstract}
Keywords: molecular biological technique; environmental engineering; microorganism; application. Abstract. With the worsening of environmental pollution issues, applications of molecular biological techniques to the field of environmental pollution treatment have also drawn wide attention. These techniques can contribute to efficient treatment of environmental pollution through microorganism, and boast the advantage of no side effects. In this paper, the author analyzes several molecular biological techniques in the field of microorganism in environmental engineering, and discusses applications of these techniques to environmental pollution treatment. It is hoped that this research can provide some references for people concerned about the issue.
\end{abstract}

\section{Introduction}

As an applied and interdisciplinary subject, Microorganism Technique has made great contributions to environmental pollution treatment. In the field of analysis of biodiversity, pollution treatment, soil restoration, odor treatment and petroleum degradation, molecular biological techniques have found wide applications. It is thus necessary to discuss about issues related to their applications to the field of microorganism in environmental engineering so as to promote development of environmental engineering.

\section{Analysis of molecular biological techniques to the field of microorganism in environmental engineering}

\subsection{PCR nucleic acid techniques}

As core techniques of molecular biological techniques, PCR nucleic acid techniques are a group of practical techniques, including the PCR-SSCP technique, the PCR-DGGE technique and the PCR-RELP technique. With the PCR-SSCP technique, DNA groups extracted from environmental samples can be efficiently analyzed. With the PCR-DGGE technique, the basic group of living materials can be tested, and DNA can be extracted from genomes of environmental samples to serve as samples for follow-up experiment. With the PCR-RELP technique, the restrictive nucleic acid incision enzyme can be used for recognition of relevant DNA, and items can be marked within materials to provide comparison reference for future tests.

\subsection{PCR sequencing techniques}

In Biology, molecular biological techniques are needed to study separation of valuable research groups or life characteristics of different categories in the microorganism environment. With PCR sequencing techniques, microbial groups of various categories and species in the microorganism environment can be confirmed to provide technical support for newly-found microbial groups. Therefore, during the biological environmental test, PCR sequencing techniques are highly practical.

2.3 Gene probing techniques

When it comes to study of microbial environment, the gene probe can be used to test specificity of the environment, and conduct sequence research of the single-stranded DNA segments. With gene probing techniques, researchers can combine the melting steps and the biological principle of the basic group structure to compare and observe interactive reaction of extracted samples [1]. The microbial gene probe can be used to label the position of the microorganism samples extracted. Based 
on that, microorganism can be compared and observed during the breeding process, and reliable technical support can be provided for microorganism studies.

\section{Applications of molecular biological techniques to the field of microorganism in environmental engineering}

3.1 Applications of molecular biological techniques to biodiversity research in the field of environmental engineering

The nature is abundant with biological species. However, most creatures observed by humans are visible to naked eyes. However, there are still lots of creatures invisible to naked eyes. For example, in rivers, soil, waste and sewage, lots of microorganism lives. With the development of the second industry, environmental pollution has been worsening, making it imperative to study biological groups in the environment and deepen humans' understanding of the environment. To the end, microorganism plays an important role in the environment. Microorganism research can lay the foundation for environmental treatment. Molecular biological techniques can be used to study microorganism diversity in the field of environmental engineering. This can better support humans' research into microorganism. For example, PCR techniques can be used to test the nitrifying bacteria in microbial communities, through which research into activated sludge can gain technical support. A specific reactor can be used to carry activated sludge with specific functions. Then, microbial communities can be extracted, and microorganism within microbial communities leading to special functions of activated sludge can be analyzed. Some foreign researchers have verified microorganism diversity in the anaerobic activated sludge, and used molecular biological techniques to reveal the reaction mechanism of different microorganisms. Through these researches, the diversity and richness of microbial communities within materials such as sludge can be better demonstrated through molecular biological techniques, which can provide vigorous technical support for development of the field of microorganism in environmental engineering.

3.2 Applications of molecular biological techniques to biodiversity research to soil restoration research in the field of environmental engineering

Excessive discharge of urban sewage has resulted in heavy metal pollution of the soil, posing a great threat to sustainable development of the human society. Soil biological restoration techniques can help restore soil, and have advantages of environmental-friendliness, low cost and in-situ restoration. Currently speaking, soil microorganism restoration techniques have become a hotspot research issue. Molecular biological techniques can be used to recognize and extract beneficial microorganism. In this way, these microorganisms can make use of their metabolism to decompose organic pollution of soil. To be specific, these microorganisms are adopted to obtain ironophores, organic acids and other materials, thus transforming heavy metals in the soil into harmless chelating states. The rhizospheric microorganisms can be used to promote plant growth, and the hyperaccumulators can contribute to soil restoration [2]. If molecular biological techniques are adopted, bacteria with a strong metabolic capability can be chosen, and the soil restoration process can been observed. In this way, the soil restoration microorganism database can be built for better understanding of the soil restoration mechanism and optimization of soil restoration techniques.

3.3 Applications of molecular biological techniques to odor treatment research in the field of environmental engineering

In real life, various treatment facilities, like the sewage treatment plant, the composting plant and the refuse landfill, can generate a large amount of odor, thus resulting in pollution of the surrounding environment. Based on the metabolism of microorganisms, the odor can be decomposed into harmless materials, including carbon dioxide, water and sulfate. Such odor treatment is efficient. With molecular biological techniques, metabolism of microorganisms during the odor treatment process can be analyzed. In this way, the deodorization of microorganisms can be enhanced through expansion of variable regions of deodorization genes of microorganisms. At the same time, molecular biological techniques can be used to analyze richness, metabolism and diversity of deodorization microorganisms. By doing so, researchers can learn how these microorganisms are restricted by 
factors including $\mathrm{pH}$ and carbon source, and screen out beneficial bacteria [3]. Therefore, molecular biological techniques can contribute to development of odor treatment research.

3.4 Applications of molecular biological techniques to sewage treatment research in the field of environmental engineering

With the development of the second industry, the industrial sewage discharge has also increased. In response to the national appeal for environmental protection, various plants are seeking efficient ways for sewage treatment so as to reduce environmental pollution of industrial production. Microbial flocculants inside and outside microbial bacteria are secreted. Biomacromolecules with electric charge can efficient remove SS and COD in domestic sewage and industrial sewage, registering a removal rate of $91 \%$ and $68 \%$, respectively. Compared with other chemicals, such as aluminum salt and ferric salt, microbial flocculants not only have stronger flocculation than activated sludge, but can help enterprises easily filter sediments. The residue obtained through the microorganism can achieve biological decomposition without causing secondary pollution to the surrounding environment [4]. Therefore, in terms of water pollution and treatment, molecular biological techniques can be employed to extract microbial flocculants, which can better treat water pollution.

3.5 Applications of molecular biological techniques to petroleum degradation research in the field of environmental engineering

In daily life, petroleum is an important resource to humans' production and life. During petroleum development and application process, environmental pollution might be easily caused. Currently speaking, petroleum pollution is one of the trickiest environmental problems. Petroleum has complex components, some of which are toxic to microorganisms. Therefore, it is necessary to identify, screen and culture microbial bacteria with a high degradation ability during petroleum pollution treatment. Only in this way can petroleum pollutants be efficiently treated. If molecular biological techniques are adopted, petroleum pollution and microorganisms can be studied deeply so as to find microorganism species conducive to petroleum pollution treatment. The use of microorganisms for petroleum degradation boasts advantages of no side effects, good environmental treatment effects and low cost [5]. In the petroleum pollution degradation research field, molecular biological techniques have been highly acclaimed by scholars and experts. Therefore, they are playing an important role during the development of the petroleum industry.

\section{Conclusions}

All in all, applications of molecular biological techniques can provide more technical support for humans' understanding, treatment and transformation of the nature, and contribute to sound development of the natural environment. Therefore, the author is convinced that, with the development of molecular biological techniques, they will get wider applications in the field of microorganism in environmental engineering.

\section{References}

[1]Shi ZH \& Wang L. Progress of applications of molecular biological techniques to the field of environmental microbiology[J]. China Science and Technology Information, 2013, 16:135+139.

[2]Li L, Zeng W, Zhang Y, et al. Application of molecular biological technology in the study of the nitrification bacteria in wastewater treatment system[J]. Journal of Henan Science and Technology, 2010, 01:135-142.

[3]Wang L, Jia W Q, Ma F, et al. Perspective of mycorrhizal technology application for environmental restoration[J]. Ecology and Environmental Sciences, 2010, 02:487-493.

[4]Qin Y, Guo J S \& Fang F. Application of molecular biology technology on microbiology study of nitrogen removal processes in wastewater treatment[J]. Science Paper Online, 2011, 05:385-389.

[5]Ma M L. Progress of microbiological analysis methods to the field of environmental engineering[J]. Science \& Technology Information, 2011, 22:183. 